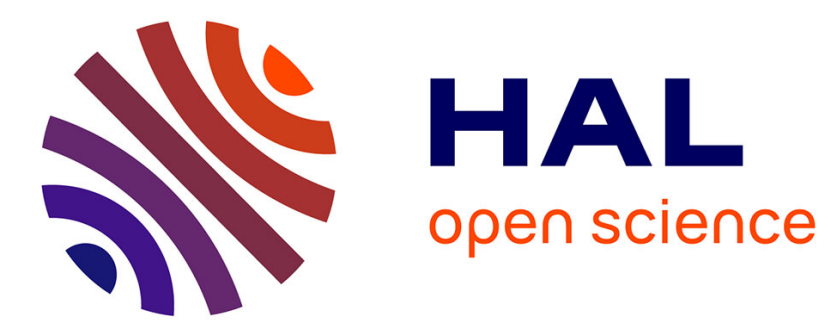

\title{
Reptation d'une chaîne hétérogène
}

P.-G. de Gennes

\section{- To cite this version:}

P.-G. de Gennes. Reptation d'une chaîne hétérogène. Journal de Physique Lettres, 1983, 44 (6), pp.225-227. 10.1051/jphyslet:01983004406022500 . jpa-00232184

\section{HAL Id: jpa-00232184 https://hal.science/jpa-00232184}

Submitted on 1 Jan 1983

HAL is a multi-disciplinary open access archive for the deposit and dissemination of scientific research documents, whether they are published or not. The documents may come from teaching and research institutions in France or abroad, or from public or private research centers.
L'archive ouverte pluridisciplinaire HAL, est destinée au dépôt et à la diffusion de documents scientifiques de niveau recherche, publiés ou non, émanant des établissements d'enseignement et de recherche français ou étrangers, des laboratoires publics ou privés. 
Classification

Physics Abstracts

$61.40 \mathrm{~K}$

\title{
Reptation d'une chaîne hétérogène
}

\author{
P. G. de Gennes
}

Collège de France, 75231 Paris Cedex 05, France

(Reçu le 24 novembre 1982, accepté le 21 janvier 1983)

\begin{abstract}
Résumé. - On discute les mouvements d'un copolymère statistique, en présence d'obstacles fixes, dans un milieu aléatoire : translation d'ensemble le long du tube ou propagation de défauts. Le temps de reptation trouvé croît exponentiellement avec la longueur de chaîne. Ces considérations seront peut-être utiles pour comprenare certaines réorganisations des protéines globulaires.
\end{abstract}

\begin{abstract}
We analyse the reptative motions of a statistical copolymer inside a random medium : overall translations and motion of "kinks ». The resulting reptation time increases exponentially with chain length. This discussion may possibly be helpful to understand certain reorganizations inside globular proteins.
\end{abstract}

1. Choix d'un modèle. - Les mouvements d'un homopolymère linéaire flexible, enchevêtré avec des obstacles fixes, sont à peu près compris [1]. Le cas d'une chaîne hétérogène est beaucoup plus complexe : certaines parties de la chaîne peuvent être piégées dans le milieu ambiant (luimême aléatoire) et se déplacer difficilement.

Nous étudions ici la situation la plus simple : une chaîne de $N$ monomères (indicés de $n=1$ à $n=N$ ) confinée dans un « tube " étroit (diamètre comparable à la taille des monomères) à l'intérieur d'un gel [2]. A chaque monomère $(n)$ est associé un indice $p_{n}\left(p_{n}=0\right.$ si le monomère est de type A et $p_{n}=1$ s'il est de type B). La moyenne $\left\langle p_{n}\right\rangle=p$ définit la composition de la chaîne. Le potentiel vu par le $n$-ième monomère a la forme

$$
U_{n}=W_{0}(i)+W_{1}(i) p_{n} .
$$

L'indice (i) spécifie la région du gel qui est en contact avec le monomère $n$. Pour une chaîne confinée dans un tube, il sera commode de définir $i$ par l'abscisse curviligne le long du tube. L'énergie totale de la chaîne sera alors

$$
V=\sum_{n=1}^{N}\left[W_{0}(n)+W_{1}(n) p_{n}\right]
$$

et si on déplace la chaîne de $s$ monomères dans le tube, $V$ devient

$$
V(s)=\sum_{n}\left[W_{0}(n+s)+W_{1}(n+s) p_{n}\right] .
$$

Dans la suite, on retranchera de ces sommes la contribution moyenne $\left\langle W_{0}\right\rangle+\left\langle W_{1}\right\rangle p$ qui donne une énergie constante. On omettra aussi la contribution $\left(W_{1}-\left\langle W_{1}\right\rangle\right) p$ qui décrit 
l'effet des fluctuations de potentiel sur une chaîne homọgène : ces fluctuations peuvent être qualitativement importantes pour une chaîne rigide mais pas pour une chaîne flexible [3]. Finalement

$$
V(s) \rightarrow \sum_{n} \delta W_{1}(n-s)\left(p_{n}-p\right)
$$

avec $\delta W_{1} \equiv W_{1}-\left\langle W_{1}\right\rangle$. Nous supposons le potentiel $\delta W_{1}$ localisé

$$
\left\langle\delta W_{1}(i) \delta W_{1}(j)\right\rangle=\delta_{i j} \delta W^{2},
$$

et aucune corrélation entre $\delta W$ et $p_{n}$. (Tous les effets de ségrégation spatiale dans le milieu sont négligés.)

2. Translations d'ensemble. - Si la chaîne se déplaçait en entier le long du tube, elle serait soumise au potentiel aléatoire $V(s)$ :

$$
\left.\begin{array}{l}
\langle V(s)\rangle=0 \quad \delta V^{2}=\left\langle V^{2}(s)\right\rangle=N \delta W^{2} p(1-p) \\
\langle V(0) V(s)\rangle=N \delta W^{2}\left\langle\delta p_{0} \delta p_{s}\right\rangle
\end{array}\right\}
$$

où $\delta p_{n}=p_{n}-p$. Les fonctions de corrélation $\langle\delta p \delta p\rangle$ sont conditionnées par le mode de synthèse chimique, mais ont une portée assez courte (quelques monomères). D'après (4) on peut se représenter le potentiel $V(s)$ comme une succession de barrières, de hauteur $\delta V \sim N^{1 / 2}$ et d'espacement donné par la portée des corrélations $\langle\delta p \delta p\rangle$. Mais cette vision est un peu sommaire pour un mouvement à une dimension : quelques barrières très hautes contrôlent la migration de la chaîne. Une approximation un peu meilleure, décrite dans la référence [4], donne un temps de migration globale

$$
T_{1} \cong D^{-1} \int_{0}^{N} \mathrm{~d} n \int_{0}^{n} \mathrm{~d} m\langle\exp [V(n)-V(m)] / k T\rangle,
$$

où $D\left(\sim N^{-1}\right)$ est un coefficient de diffusion sans interactions. Le potentiel $V(n)-V(m)$ a une distribution gaussienne : en calculant alors la moyenne dans (5), on aboutit à une forme exponentielle $T_{1}=T_{0}(N) \mathrm{e}^{N \alpha}$

$$
\alpha=N^{-1}(\delta V / k T)^{2}=\left(\frac{\delta W}{k T}\right)^{2} p(1-p) .
$$

Le préfacteur $T_{0}(N)$ est ici d'ordre $N^{2} D^{-1} \sim N^{3}$ mais ces préfacteurs sont peu importants pour notre discussion.

3. Mouvement d'un défaut. - En réalité, la chaîne ne progresse pas dans le tube par translation d'ensemble, mais plutôt par déplacement de "défauts » localisés («kinks ») [1]. Quand un tel défaut traverse un monomère donné sur la séquence chimique, il translate ce monomère le long du tube par une distance $b$ égale à sa "longueur stockée ». Ici pour simplifier, nous prendrons $b=a$ (taille du monomère) : donc le passage de 1 défaut d'un bout à l'autre de la séquence chimique décale toute la chaîne de une unité. Si un tel défaut se déplace de $n$ à $n+s$ sur la séquence chimique, il modifie l'énergie de la chaîne par la quantité

$$
V_{\mathrm{d}}(s)=\sum_{m=n}^{m=n+s}\left(\delta W_{m+1}-\delta W_{m}\right)\left(p_{m}-p\right) .
$$


Pour $s \gg 1$, le potentiel $V_{\mathrm{d}}$ est ici encore gaussien, de moyenne nulle, avec

$$
\left\langle V_{\mathrm{d}}^{2}(s)\right\rangle=2 \delta W^{2} s\left[p(1-p)-\left\langle\delta p_{0} \delta p_{1}\right\rangle\right] \text {. }
$$

En revenant à une formule de type (5) on trouve un temps de diffusion pour un défaut allant d'un bout à l'autre de la chaîne :

$$
\begin{aligned}
\tau_{1} & =\tau \exp \left(N \alpha_{\mathrm{D}}\right) \\
\alpha_{\mathrm{D}} & =\left(\frac{\delta W}{k T}\right)^{2}\left[p(1-p)-\left\langle\delta p_{0} \delta p_{1}\right\rangle\right] .
\end{aligned}
$$

$\alpha_{\mathrm{D}}$ est plus petit que $\alpha$ s'il existe des corrélations positives $\left\langle\delta p_{0} \delta p_{1}\right\rangle$. Le temps global de reptation $\tau_{\text {rep. }}$ pour la chaîne est le temps requis pour faire passer $N$ défauts d'un bout à l'autre. Il dépend de la concentration à l'équilibre des défauts; mais ces considérations n'influent que sur le préfacteur

$$
T_{\text {rep. }}(\delta W)=T_{\text {rep. }}(0) \exp \left(N \alpha_{\mathrm{D}}\right) .
$$

4. Discussion. - Le cas du gel très réticulé, à tube étroit, que nous avons choisi, se prête mal à l'expérimentation. Pour des gels plus lâches, gonflés par un solvent, il faudrait reprendre l'analyse : diviser la chaîne en sous-unités de longueur comparable à celle $\left(N_{\mathrm{e}}\right)$ des maillons du gel. Les fluctuations à l'échelle $N_{\mathrm{e}}$ sont très réduites, et les effets décrits plus haut doivent disparaître dès que $N_{\mathrm{e}} \gg 1$. Ceci est probablement le cas pour les copolymères statistiques fondus. Ici chaque chaîne eșt enfermée dans un "pseudogel " et $N_{\mathrm{e}}$ (distance entre enchevêtrements) est grand $(\sim 300)$. Un cas intéressant mais plus complexe serait celui des réorganisations d'une protéine globulaire (débuts de dénaturation, transformations allostériques, ...). Ici, on peut peut-être penser en termes de reptation par " kinks ", même si la structure est localement rigide. Le milieu ambiant est en fait la même chaîne polypeptidique, et n'est donc pas totalement fixe. Il y a souvent une certaine ségrégation spatiale entre régions hydrophiles et hydrophobes, qui donnerait des corrélations entre $\delta W$ et $\delta p$. Mais, si on néglige ces complications, on est conduit à une observation intéressante. Supposons que la chaîne soit aspirée à un bout par une force de succion constante $\phi$. Alors, l'analyse de la référence [4] nous suggère qu'il y a deux régimes : a) pour $\phi$ faible, une sorte de fluage logarithmique (lié au temps exponentiel de l'Eq. (10)); $b$ ) pour $\phi$ fort, un glissement rapide. Ces considérations seront peut-être utiles un jour pour comprendre certaines étapes de construction sur un ribosome.

\section{Bibliographie}

[1] DE Gennes, P. G., J. Chem. Phys. 55 (1971) 572.

DE Gennes, P. G. et Léger, L., Ann. Rev. Phys. Chem. 33 (1982) 49.

[2] Le cas d'une chaîne homogène, non confinée dans un tube, et soumise à des potentiels aléatoires, a été discuté récemment par G. C. Martinez-Mekler et M. A. Moore, J. Physique Lett. 42 (1981) L-413. Ce cas est compliqué, car le potentiel doit avoir deux fonctions distinctes : engendrer un " tube " et moduler le mouvement dans le tube. (Il n'est pas sûr que le tube décrit dans cette référence ait un diamètre indépendant du temps.)

[3] DE GenNes, P. G., C.R. Heb. Séan. Acad. Sci 294B (1982) 827.

[4] DE Gennes, P. G., J. Stat. Phys. 12 (1975) 463. 